\title{
Spatial Reconstruction of Atom Probe Data from Zircon
}

\author{
D.W. Saxey ${ }^{1 *}$, D. Fougerouse ${ }^{1,2}$, W.D.A. Rickard ${ }^{1}$ and S.M. Reddy ${ }^{1,2}$ \\ 1. Geoscience Atom Probe, Advanced Resource Characterisation Facility, John de Laeter Centre, Curtin \\ University, Perth, Australia. \\ 2. School of Earth and Planetary Science, Curtin University, Perth, Australia. \\ * Corresponding author: david.saxey@curtin.edu.au
}

The importance of zircon $\left(\mathrm{ZrSiO}_{4}\right)$ as a source of geological information at sub-micron scales [1-3] has driven significant interest in the optimization of its analysis by atom probe tomography (APT) [4-6]. Here we report a set of atom probe experiments, using the reference material 91500 [7], aimed primarily at informing a 'good' spatial reconstruction of zircon data. As crystallographic features are typically absent from APT data on silicate minerals, the reconstruction cannot be calibrated using methods that depend on lattice information. Similarly, nanoscale features are not always present or available for accurate imaging, which may otherwise allow calibration by correlative methods.

For many geological applications, producing a highly-accurate spatial reconstruction is not essential, and a voltage-based reconstruction, in which the instantaneous tip radius scales with the specimen voltage, is usually adequate. The reconstruction problem is then reduced to an appropriate selection of parameters for this reconstruction algorithm [8]. In this study, the Cameca Integrated Visualization and Analysis Software (IVAS 3.6.12) was used to reconstruct the data with the following default settings: image compression factor $=1.65$, k-factor $=3.3$, detector efficiency $=0.36$. The atomic density of zircon $(\mathrm{n})$ in atoms $/ \mathrm{nm}^{3}$ can be calculated using the density $(\rho=4.71)$ in $\mathrm{g} / \mathrm{cm}^{3}$, the molecular weight of the $\mathrm{ZrSiO}_{4}$ formula unit $(\mathrm{M}=183.3)$ in $\mathrm{g} / \mathrm{mol}$, and the number of atoms making up a single formula unit $(\mathrm{z}=6)$ : $\underline{\mathrm{n}=\mathrm{z} \rho \mathrm{N}_{\mathrm{A}}} /\left(\mathrm{M} \mathrm{x} \mathrm{10^{21 } )}\right.$, where $\mathrm{N}_{\mathrm{A}}$ is Avogadro's Number. This equates to 92.9 atoms $/ \mathrm{nm}^{3}$, and is significantly different from the value often provided automatically by the IVAS software (43.9 atoms $/ \mathrm{nm}^{3}$ ), which corresponds to metallic zirconium. Fixing a calculated density for zircon leaves only the evaporation field to be chosen for a complete voltage-based reconstruction.

Atom probe specimens were prepared from a single crystal of the 91500 reference material using conventional lift-out and focused ion beam milling techniques in a Tescan Lyra 3 FIB-SEM, with small differences deliberately introduced between the needle shapes. The specimens were field-evaporated in the atom probe (Cameca LEAP 4000X HR), removing 1 - 5 million ions at a base temperature of $50 \mathrm{~K}$, using a $300 \mathrm{pJ}$ laser pulse energy and an ion detection rate of 0.01 . High-resolution secondary electron (SE) images were obtained from each specimen, using a pre-titled sample holder to align the needle axis perpendicular to the electron beam. Once imaged, each specimen was field-evaporated in a long atom probe acquisition, recording between 35 and 60 million ions. Post-run SE imaging was arranged to view each tip from the same angle and magnification, to allow direct comparison with the pre-run images.

Pre- and post-acquisition images were overlaid to determine the depth of material removed (Fig 1). Eight specimens were analysed with this method in order to explore the variability that may occur due to subtle differences in specimen geometry or tip alignment, for example. The eight reconstructed z-depths were fitted to the SE observations using linear regression and adjusting the evaporation field (Fig 2). The best fit yielded an evaporation field of $32.2 \pm 0.3 \mathrm{~V} / \mathrm{nm}$ for an atomic density of 92.9 atoms $/ \mathrm{nm}$. Using this approach has produced spatial reconstructions in good agreement with correlative methods [9]. 


\section{References:}

[1] JW Valley et al., Nature Geoscience 7(3) (2014), p. 219.

[2] SM Reddy et al., Geochimica et Cosmochimica Acta 195 (2016), p. 158.

[3] SD Montalvo et al., Chemical Geology 507 (2019), p. 85.

[4] A La Fontaine et al., Microsc. Microanal. 23(2) (2017), p. 404.

[5] DW Saxey et al. in "Microstructural Geochronology: Planetary Records Down to Atom Scale", ed. D Moser et al., (AGU/Wiley Publishing) p.293.

[6] F Exertier et al., Chemical Geology 495 (2018), p. 27.

[7] M Wiedenbeck et al., Geostandards and Geoanalytical Research 28 (2004), p. 9.

[8] B Gault et al., Ultramicroscopy 111 (2011), p. 448.

[9] EM Peterman et al., Science Advances 2 (2016), e1601318.
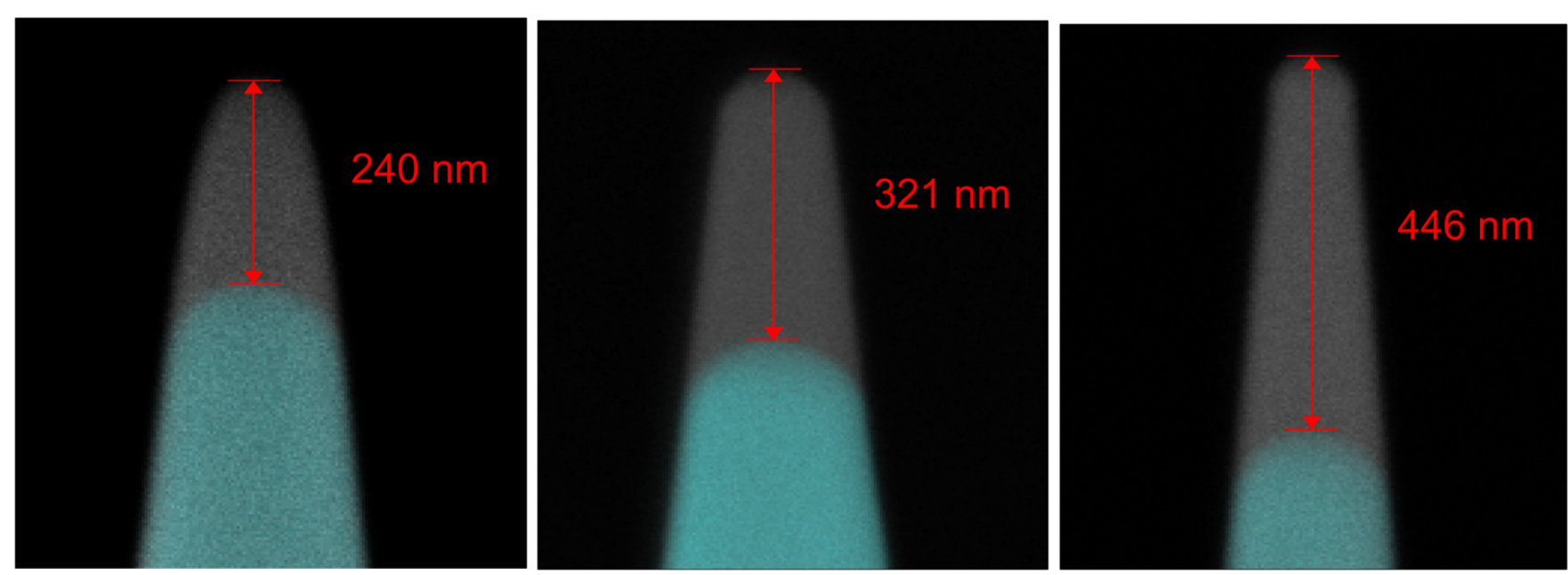

Figure 1. Overlaid images of zircon specimens before (grey) and after (green) atom probe data acquisition.

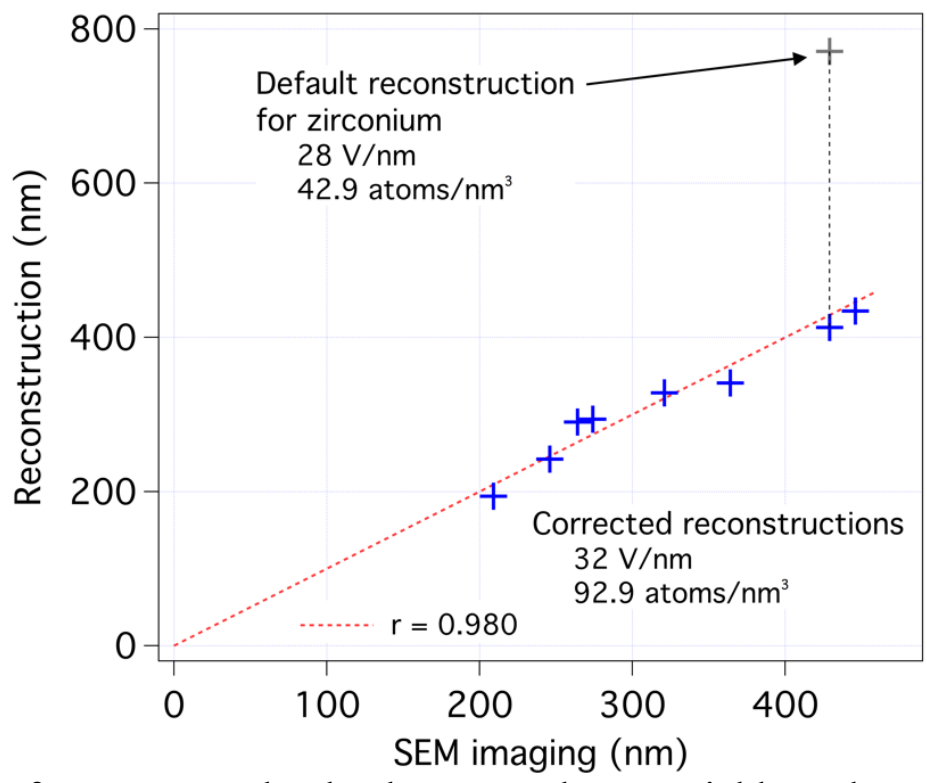

Figure 2. Scatter plot of reconstructed z-depths versus the material loss along the specimen axis observed by SE imaging. Using the default parameters for zirconium yields an inaccurate reconstruction. 